\title{
Tachykinin neuropeptides in cerebellar granule neurons: an immunocytochemical study
}

\author{
C. Barbato, ${ }^{* \wedge}$ M.T. Ciotti, ${ }^{*}$ A. Serafino, ${ }^{*}$ C. Severini, ${ }^{*}$ P. Calissano*^ \\ *Istituto di Neurobiologia e Medicina Molecolare, CNR, Roma, Italia; ^Dipartimento di Neuroscienze, \\ Università di Roma Tor Vergata, Roma, Italy
}

(C)2005, European Journal of Histochemistry

We previously demonstrated that exogenously administered neurokinin $A$ and neurokinin $B$, but not substance $P$, increased the sensitivity of cultured cerebellar granule neurons (CGNs) to glutamate. In the present study, the presence of tachykinin neuropeptides in CGNs was tested by confocalbased immunofluorescence. We found that neurokinin $A$ and neurokinin $B$ are present in CGNs but absent in astrocytes. while substance $P$ is abundant in astrocytes but absent in CGNs. It is postulated that the different localization of tachykinin neuropeptides in CGNs and astroglial cells has a physiological role in the modulation of excitatory transmission.

Key words: Tachykinin, neurokinin A, neurokinin B, substance $\mathrm{P}$, cerebellar granule neurons

Correspondence to: Prof. Pietro Calissano

Istituto di Neurobiologia e Medicina Molecolare, CNR

Viale Marx 15/43, I-00137, Roma, Italy

Tel.: +39.0686090248

Fax.: +39.0686090370

E-mail: calissano@in.rm.cnr.it

Paper accepted on October 15, 2004

European Journal of Histochemistry 2005; vol. 49 issue 1 (Jan-Mar):???-???
T achykinin neuropeptides (TKs), which include substance $P(S P)$, neurokinin $A(N K A)$, and neurokinin $B(N K B)$, interact with three distinct types of surface $\mathrm{G}$-protein-coupled receptors called respectively, NK1, NK2 and NK3 (Nakanishi et al., 1990; Maggi et al., 1993; Mussap et al., 1993; Buch 1994). TKs are widely distributed both in the central and peripheral nervous systems of mammals where they exert a variety of effects in physiological and pathological conditions (Helke et al., 1990, Raffa 1998, Stumm et al., 2001, Severini et al., 2002). The distribution of TKs has been extensively studied by molecular, biochemical, and immunohistochemical techniques (Tsuchida et al., 1990). SP and NKA are usually co-expressed and are found in high concentrations in both the peripheral and central nervous systems, whereas NKB is confined mostly to the central nervous system (Tsuchida et al., 1990, Otsuka et al., 1993). TKs have also been demonstrated in the rat cerebellum where the amount of NKA and NKB is reported to be three times lower than that of SP (Otsuka et al., 1993). We have recently demonstrated that NKA and $N K B$, but not $S P$, increase the response of cultured cerebellar granule neurons (CGNs) to the excitotoxic action of glutamate, suggesting that the former exert an upregulating action on the glutamatergic system (Severini et al., 2003) and further suggesting that NK2 and NK3 receptor-preferring peptides operate in an autocrine or paracrine fashion. A glutamate upmodulating function, probably via diffusion transmission, has previously been hypothesized for TKs acting in rat neocortex (Kaneko et al., 1998). This hypothesis suggests the presence of these peptides, and not only of their receptors as previously reported, in the same cultured CGNs. In order to evaluate the possible presence of TK neuropeptides in CGNs, we carried out an investigation by means of confocal microscopy immunofluorescence analysis. We found that both NKA and NKB are present in CGNs while they are absent in the astroglial primary cultures. On the contrary, the astrocytes contain SP which is absent in the CGNs. 


\section{Materials and Methods}

\section{Neuronal cultures}

Cultures enriched in granule neurons were obtained from dissociated cerebella of 8-day-old Wistar rats (Charles River, Calco, Italy) (Levi et al., 1984). Cells were plated in basal medium Eagle (BME; Life Technologies, Gaithersburg, MD) supplemented with $10 \%$ heat-inactivated fetal bovine serum (FBS), $25 \mathrm{mM} \mathrm{KCl}$, and $2 \mathrm{mM}$

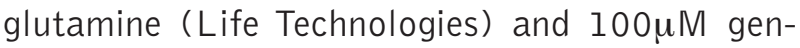
tamicin sulfate, on dishes with coverslips (Nunc, Roskilde, Denmark) coated with poly-L-lysine. Cells were plated at $2.5 \times 10^{6}$ cells per $35 \mathrm{~mm}$ dish. $1 \beta$-Arabinofuranosylcytosine $(10 \mathrm{mM})$ was added to the culture medium 18-22 hr after plating to prevent proliferation of non-neuronal cells. Astroglial primary cultures were prepared as described for granule cells, with the following modification: cells were plated at low density ( $3 \times 10^{6}$ cells per $90 \mathrm{~mm}$ dish with coverslips) and cultured in BME supplemented with $10 \%$ heatinactivated $\mathrm{FBS}$ and $5 \mathrm{mM} \mathrm{KCl}$.

\section{Immunofluorescence}

CGNs and astroglial cells were fixed and permeabilized for NKB with 3.5\% formaldehyde, 70\% ethanol and 5\% acetic acid for $15 \mathrm{~min}$ at room temperature. For NKA and SP immunofluorescence, the neuronal cells were fixed with $4 \%(\mathrm{w} / \mathrm{v}$ in $\mathrm{PBS}$ ) paraformaldehyde for $15 \mathrm{~min}$ at room temperature. Fixed cells were washed in $\mathrm{PBS}, \mathrm{pH}$ 7.4, and permeabilized with $0.2 \%$ Triton $\mathrm{X}-100$ Tris $\mathrm{HCl}, \mathrm{pH} 7.4$, for $5 \mathrm{~min}$. The coverslips were treated with rabbit polyclonal antibodies against NKB 1:100, NKA 1:100 (Phoenix Pharmaceuticals, Inc) and goat polyclonal antibody anti-SP 1:100 (Santa Cruz Biotechnology) or with monoclonal antibody to glial fibrillary acid protein (GFAP) 1:100 (Sigma), a specific marker for glial cells, for $1 \mathrm{hr}$ at room temperature in a moist chamber, rinsed in PBS, and stained with FITC or TRITC conjugated secondary antibodies (Sigma) for $30 \mathrm{~min}$. In order to assess the specificity of the antibody employed, rabbit anti-neurokinin $B$ serum was preincubated with $10 \mu \mathrm{g}$ of NKB peptide overnight at $4^{\circ} \mathrm{C}$ before incubation with CGNs. Alternatively, the rabbit anti-neurokinin A serum was preincubated with $10 \mu \mathrm{g}$ of NKA or NKB peptide overnight at $4^{\circ} \mathrm{C}$ and the immunofluorescence on neuronal cells was detected.

\section{Confocal laser scanning microscopy}

Fluorescently labeled preparations were observed by a confocal fluorescent imaging system using the confocal laser scanning microscope LEICA TCS 4D (Leach Instruments, Heidelberg, Germany) supplemented with an argon/krypton laser and equipped with 40x1.00-0.5 and 100x1.3-0.6 oil immersion lenses. The excitation/emission wavelengths employed were $488 \mathrm{~nm} / 510 \mathrm{~nm}$ for FITC-labelling, and $568 \mathrm{~nm} / 590 \mathrm{~nm}$ for TRITC-labelling. In order to visualize the localization of NKs in both cell body and neurites, confocal sections were taken at intervals of $0.5 \mu \mathrm{m}$ from the middle to the bottom toward the cells and a 3D reconstruction image of fluorescent signal was obtained. In double staining of TKs, confocal sections for both fluorescent signals were taken simultaneously, the $3 \mathrm{D}$ reconstruction images were recorded, and merged images of the two signals were obtained using confocal microscope software.

\section{Results}

As assessed by confocal microscopy analysis using polyclonal antibodies directed against $N K B$, NKA and SP, a distinct NKB immunoreactivity signal was clearly evident in the perikaryon of CGNs while axons and dendrites were barely visible (Figure 1A). All cultured neurons were positive for NKB with a typical punctate distribution throughout the cytoplasm (Figure 1A). No staining was detectable when a secondary antibody alone was used (data not shown).

As shown in Figure $1 B$, preabsorption with $N K B$ abolished the CGNs staining, demonstrating the specificity of the rabbit serum. Immunoreactivity to NKA was analysed using an anti-NKA rabbit polyclonal antibody. In this case, an intense staining was detected in the neuronal cell body and throughout dendrites and neurites of about $83 \%$ of the cell population (Figure 2A). As for neurokinin B, no staining was visible when the immunofluorescence was carried out with the secondary antibody alone (data not shown). Since selective NKA antibodies are not commercially available, we used in this study an antiserum for NKA ( $100 \%$ activity) which cross-reacts with NKB ( $80 \%$ activity) but not with $\mathrm{SP}$, as indicated by the manufacturer. After the preabsorption of the rabbit anti-NKA serum with NKA peptide, the staining was only partially reduced, confirming the weak specificity of the antibody. 


\section{NEUROKININ B}
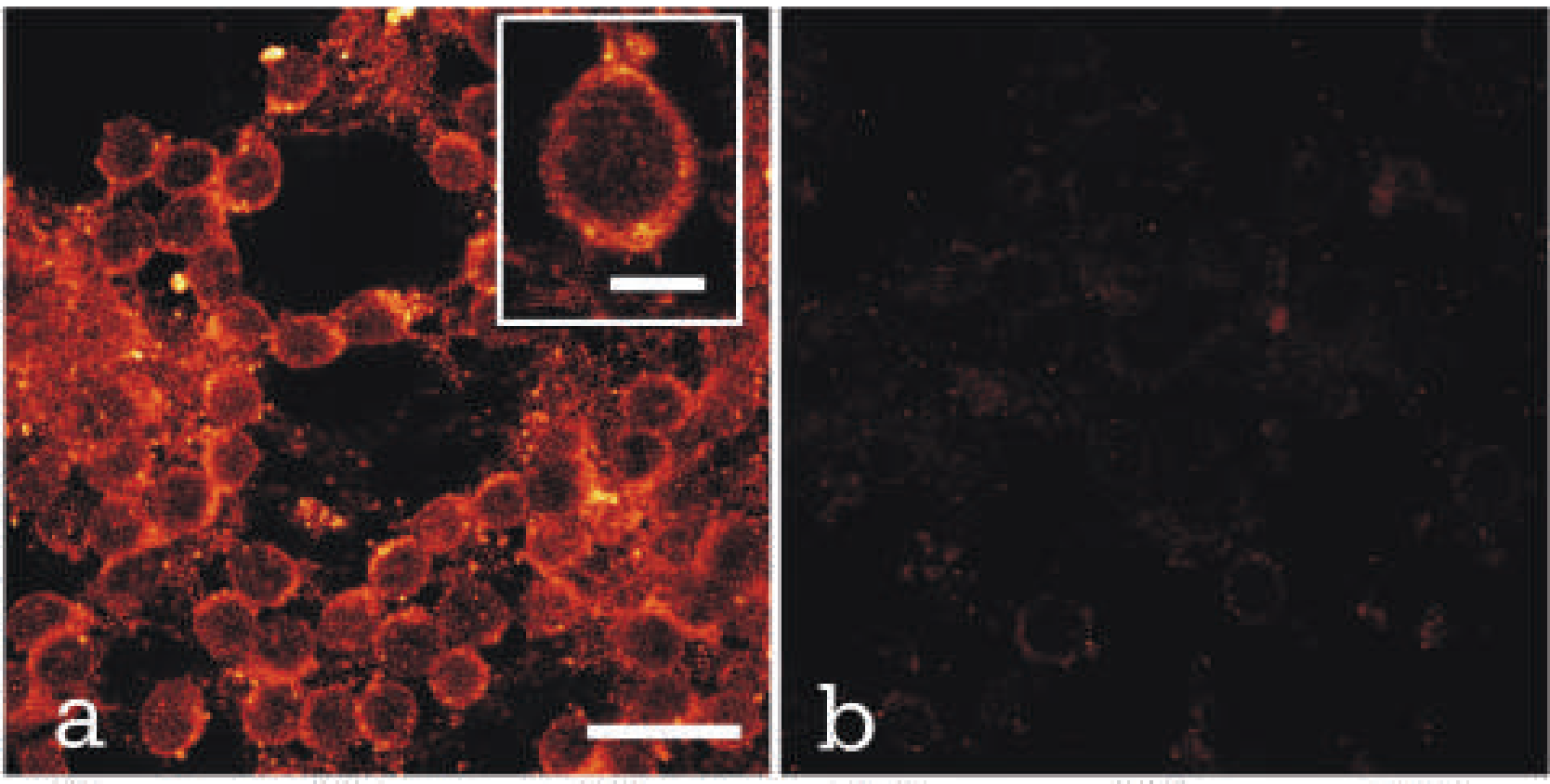

0

Figure 1. Confocal immunofluorescence of NKB in rat cerebellar granule cells culture. A: after 6 days in vitro, the CGNs were stained with a rabbit anti-NKB serum, and B: after preincubation of anti-NKB with $10 \mu \mathrm{g}$ of NKB peptide. Scale bar $20 \mu$; Corner $5 \mu$.The color bar (bottom) represents increasing fluorescence intensity.

\section{NEUROKININ A}

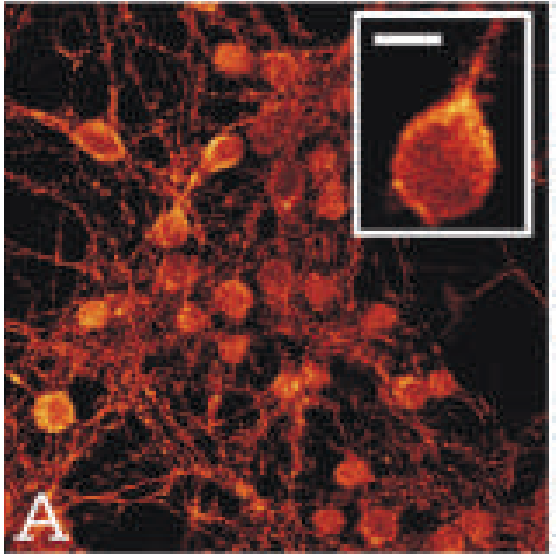

0

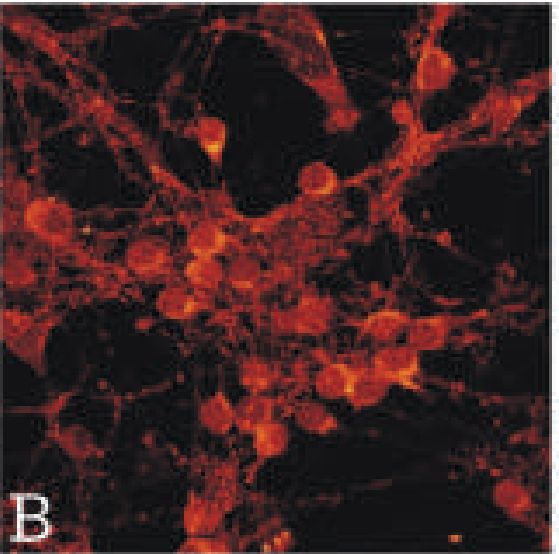

150

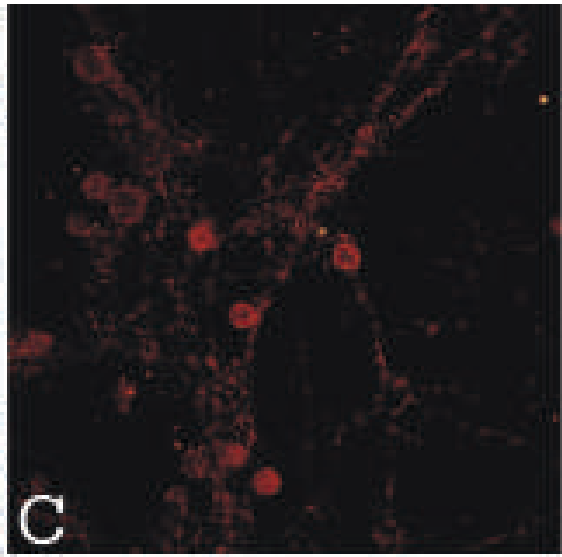

200

Figure 2.Confocal immunofluorescence of NKA in rat CGN culture. A: after 6 days in vitro, the CGNs were stained with a rabbit antineurokinin A serum, and B: after and preincubation of anti-NKA with $10 \mu \mathrm{g}$ NKA peptide and C with $10 \mu \mathrm{g}$ NKB peptide. Scale bar 20 $\mu$; Corner $5 \mu$. The color bar (bottom) represents increasing fluorescence intensity. 
Therefore, the anti-NKA antibody was preincubated with NKB peptide in order to confirm the cross-reactivity with this neuropeptide. In this case, the remaining anti-NKA immunoreactivity (Figure $2 \mathrm{C}$ ), was moderately intense with respect to the control immunofluorescence (Figure 2A), maintaining a light signal on cell processes and indicating a NKA localization in cell bodies under these experimental conditions. To determine the presence of SP in CGNs, an immunofluorescence confocal microscopy analysis was performed using a selective goat polyclonal antibody anti-SP. As shown in Figure $3 A-B$, the SP immunoreactivity was absent despite the use of two different fixation and permeabilization protocols. As $2-5 \%$ of astroglial cells are normally present in 8-days postnatal rat cerebellum cultures (Bignami et al., 1972, Eng et al., 1982), we analysed TKs immunoreactivity in this cell population by immunofluorescence. Cultures enriched with astrocytes were prepared as described in the Methods, and the immunofluorescence analysis was carried out employing the same anti-NKB, anti-NKA and anti-SP antibodies used for TKs detection in CGNs (Figure $4 A 1, B 1, C 1$ ). Doubleimmunostaining with these antibodies and with anti-GFAP (Figure 4A2; B2) showed the absence of immunoreactivity to NKA and NKB as evidenced by merge analysis (Figure 4A3, B3). On the contrary, when astrocytes were stained with the same anti-SP goat polyclonal antibody employed in CGNs, an intense and diffuse signal was observed (Figure 4C1-C3).

\section{Discussion}

We have recently demonstrated that only NK2 and $N K 3$ receptor-preferring $T K s$, such as NKA and $N K B$ can increase the glutamate response of CGNs while NKI receptor selective agonists (such as SP) do not affect their response to glutamate, suggesting a TKs up-regulating action on this system (Severini et al., 2003). While the presence of TKs has been demonstrated by molecular, biochemical and immunohistochemical techniques in the intact rat cerebellum (Otsuka et al., 1993), no data are available at present on the distribution of such peptides in cultured CGNs. In this study, we demonstrate by immunofluorescence techniques the presence of TKs in cerebellar cultures. We found that, in CGNs, only NKB and NKA but not SP are present, while in cerebellar astrocytes only SP is detectable. This different cellular distribution was obtained using the same anti-TKs antibodies and different fixation and permeabilization protocols. In CGNs, while NKB immunoreactivity was confined to the perikaryon of $100 \%$ of neurons tested, NKA showed an intense staining both in the neuronal cell body and in neurites of $83 \%$ of the population. As described above, the staining performed with the anti-NKA antibody indicates a strong cross-reactivity with NKB peptide, but after anti-NKA antibody pre-incubation with NKB peptide, we observed only a residual fluorescent signal on cell bodies and cell processes. In all experiments performed on CGNs, we observed only a NKB localization in the neuronal cell body, whereas the NKA signal also

\section{SUBSTANCE $P$}

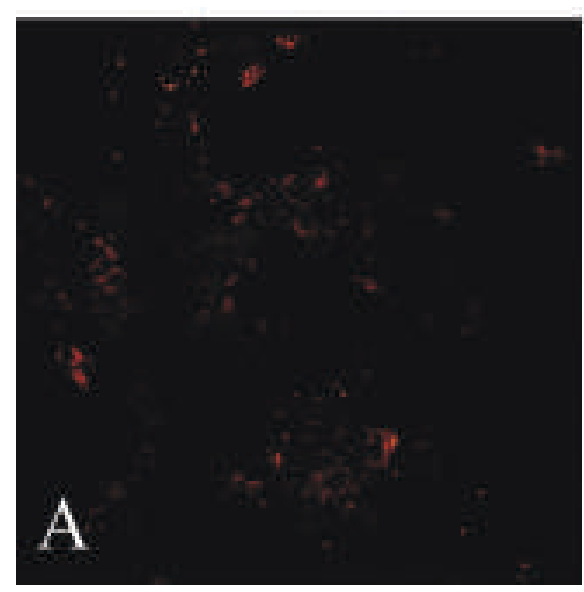

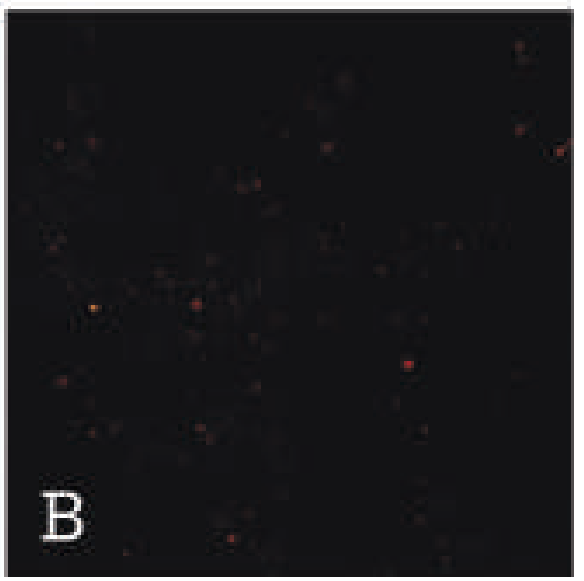

Figure 3. Confocal immunofluorescence of SP in rat CGN culture. $A$ and B: after 6 DIV, the CGNs were stained with a goat polyclonal anti-SP antibody using procedures as described in the text. Two representive immunofluorescence images are shown. 

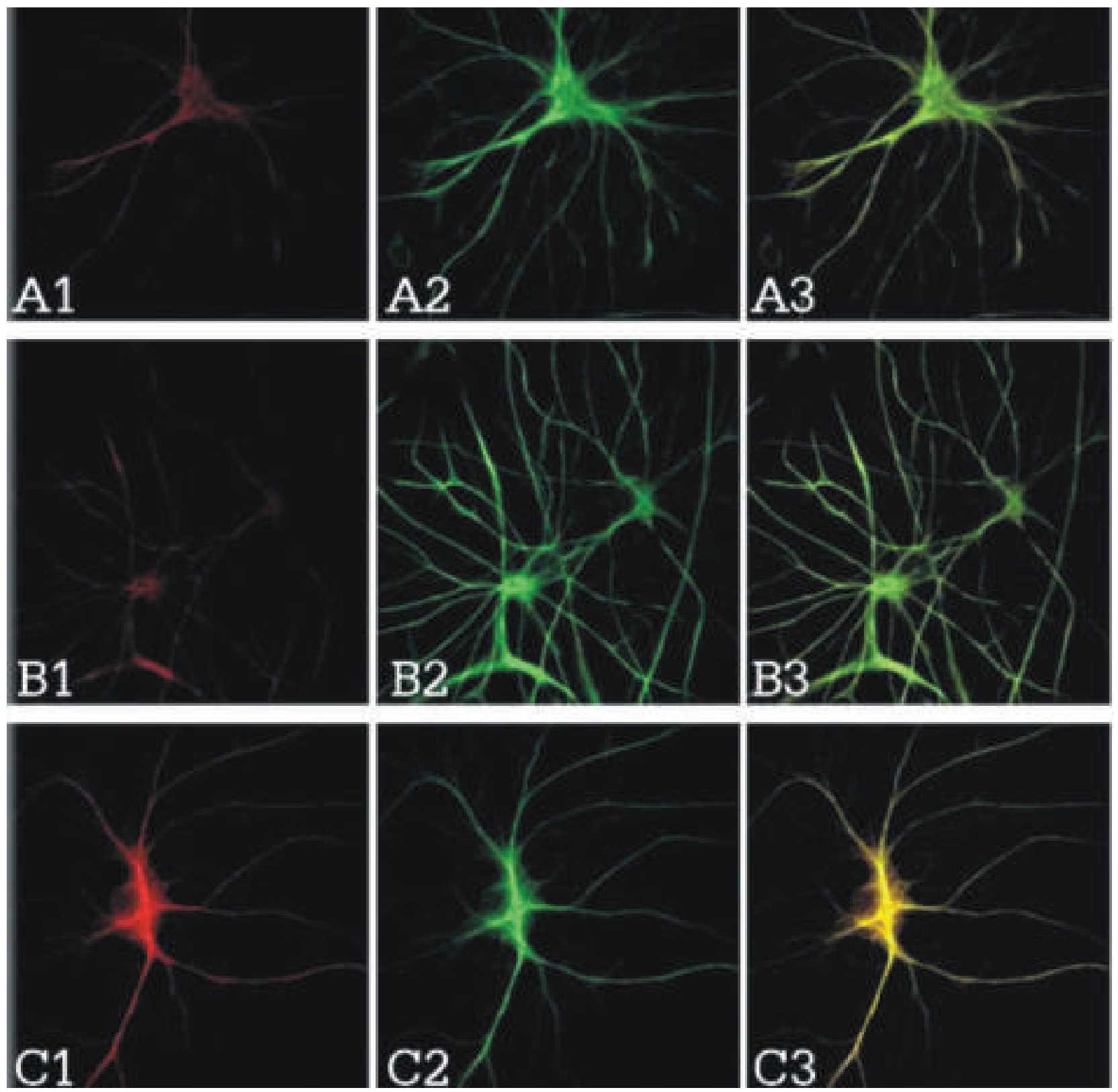

Figure 4. Confocal immunofluorescence of TKs in astroglial cell culture. A1,B1 and -C1: cultures enriched in astrocytes (see Materials and Methods) were stained with anti-NKB anti-NKA and SP, respectively, and A2, B2 and C2: with a monoclonal antibody to GFAP. A3, B3 and C3: merge analysis with anti-GFAP, shows a yellow signal only for SP.

depicted cell processes. Previous report have shown that the NKA and NKB precursors preprotachykinins $A$ and $B$, were present in rat cerebral cortex, and the immunoreactivity was observed mainly in the cell body of cortical neurons (Kaneko et al., 1998). However, it has been reported that terminals containing TKs often lack synaptic structures, suggesting a non-synaptic model of release and sites of transmitter action in addition to synaptic models (Kaneko et al., 1998; Otsuka et al., 1993). In addition, in vivo studies in rats have shown the possibility that $\mathrm{TKs}$, released by the cell body may act as local growth factors, increasing the number of synapses between the Purkinje cells and the parallel fibers (Baloyannis et al., 2000). The present data, demonstrating the presence of NKA and NKB in CGNs, reinforce the hypothesis that TKs can exert a physiological function in vivo, as shown by exogenously administered NKA and $N K B$, which increase the sensitivity of rat CGNs to glutamate (Severini et al., 2003). On the other hand, SP immunoreactivity was present only in 
astroglial cells, whereas NKA and NKB immunoreactivity was not detectable. Moreover, we identified the presence of NKB and NKA immunoreactivity in the cerebellar microglia (data not shown), indicating that these cells are probably a source of TKs other than SP, as recently reported (Lai et al., 2000). The role of such a reciprocal localization of TKs in CGNs, astroglia and microglia might be related to an autocrine or paracrine modulation of excitatory transmission affecting a variety of physiological and pathological conditions, as indicated by evidence suggesting intrinsic neuroprotective and neurodegenerative properties of these neuropeptides ( $R$ affa, 1998). In conclusion, in addition to the neuropharmacological characterization of TK neuropeptides in cultured CGNs (Severini et al., 2003), this work characterizes these TKs immunohistochemically in the cultured CGNs

\section{Acknowledgements}

This work was supported by a Ministero della Salute grant Progetto Strategico sulla malattia di Alzheimer and by MIUR-PRIN 2003 to PC. We thank Prof. T. Renda and Prof. L. D'Este for helpful discussions and suggestions during the course of this work.

\section{References}

Bignami A, Eng L, Dalhl L, Uyeda C. Localization of the glial fibrillary acidic protein in astrocytes by immunofluorescence. Brain Res 1972; 43:429-35.

Baloyannis SJ, Costa V, Deretzi G, Michmizos D. Intraventricular administration of substance $P$ increases the dendritic arborisation and the synaptic surfaces of Purkinje cells in rat's cerebellum. Int J Neurosci 2000; 101:89-107.

Buch S. H. The Tachykinin Receptors. Clifton, NJ: Humana Press, 1994.

Eng L, De Armond S. Immunocytochemical studies of astrocytes in normal development and disease. Advanc Cell Neurobiol 1982; 3:145-54.

Helke CJ, Krause JE, Manthy PW, Couture R, Bannon M.J. Diversity in mammalian tachykinin peptidergic neurons: multiple peptides, receptors, and regulatory mechanisms. FASEB J 1990; 4 :160615.

Kaneko T, Murashima M, Lee T, Mizuno N. Characterization of neocortical non-pyramidal neurons expressing preprotachykinins $A$ and B: a double immunofluorescence study in the rat. Neuroscience 1998; 86:765-81.

Lai JP, Zhan GX, Campbell DE, Douglas SD, Ho W.Z. Detection of substance $P$ and its receptor in human fetal microglia. Neuroscience 2000; 101:1137-44.

Levi G, Aloisi F, Ciotti MT, Gallo V. Autoradiographic localization and depolarization-induced release of acidic amino acids in differentiating granule cultures. Brain Res 1984; 290:77-86.

Maggi CA, Patacchini R, Rovero P, Giachetti A. Tachykinin receptors and tachykinin receptor antagonists. J Auton Pharmacol 1993; 13: 23-93.
Mussap CJ, Geraghty DP, Burcher E. Tachykinin receptors: a radioligand binding perspective, J Neurochem 1993; 60:1987-2009.

Nakanishi S, Ohkubo H, Kakizuka A, Yokota Y, Shigemoto R, Sasai Y. Molecular characterization of mammalian tachykinins receptor and a possible epithelial potassium channel. Recent Prog Horm Res 1990; 46: 59-84.

Otsuka M, Yoshioka K. Neurotransmitter functions of mammalian tachykinins. Physiological Reviews 1993; 73:229-307.

Raffa R.B. Possible role(s) of neurokinins in CNS development and neurodegenerative or other disorders. Neurosci Biohehav Rev 1998; 22:789-813.

Severini C, Improta G, Falconieri-Erspamer G, Salvatori S, Erspamer V. The tachykinin peptide family. Pharm Rev 2002; 54:1-38.

Severini C, Ciotti MT, Mercanti D, Barbato C, Calissano P. A tachykinin-like factor increases glutamate toxicity in rat cerebellar granule cells. Neuropharmacology 2003; 44:117-24.

Stumm RK, Culmsee C, Schafer MKH, Krieglstein J, Weihe E. Adaptative plasticity in tachykinin and tachykinin receptor expression after focal cerebral ischemia is differentially linked to GABAergic and glutamatergic cerebrocortical circuits and cerebrovenular endothelium. J Neurosci 2001; 21:798-811.

Tsuchida K, Shigemoto R, Yokota Y, Nakanishi S. Tissue distribution and quantitation of the $\mathrm{mRNAs}$ for three rat tachykinin receptors. Eur J Biochem 1990; 193:751-757. 